\title{
Photocount statistics of chaotic lasers
}

\author{
G. Hackenbroich, C. Viviescas, B. Elattari, and F. Haake \\ Universität Essen, Fachbereich 7, 45117 Essen, Germany
}

We derive the photocount statistics of the radiation emitted from a chaotic laser resonator in the regime of single-mode lasing. Random spatial variations of the resonator eigenfunctions lead to strong mode-to-mode fluctuations of the laser emission. The distribution of the mean photocount over an ensemble of modes changes qualitatively at the lasing transition, and displays up to three peaks above the lasing threshold.

PACS numbers: 42.50.Ar, 05.45.-a, 42.25.Dd, 42.55.-f

Nonlinear interactions in active optical media can drastically affect the statistics of the photon field. The textbook example is the single-mode laser [1.2]: Below the laser threshold, losses outweigh the gain by linear amplification such that nonlinear saturation is negligible. Then the photon statistics is well represented by a thermal distribution, and the probability $P_{n}$ for the occupation of the mode with $n$ photons decays as the power law $P_{n} \sim[\bar{n} /(1+\bar{n})]^{n}$ ( $\bar{n}$ is the mean photon number). Above threshold nonlinear interactions stabilize the field intensity. The (relative) intensity fluctuations are strongly reduced below the value found for a thermal distribution, and far above threshold $P_{n}$ approaches the Poissonian distribution $P_{n}=\left[\bar{n}^{n} / n !\right] \exp (-\bar{n})$ characteristic for a coherent state. These facts have been known since the development of the quantum theory of lasing in the 1960s.

Interest in the photon statistics of amplifying media was recently renewed by experiments on artificially fabricated random media. In random media radiation is scattered in an irregular, chaotic way. Practical realizations include laser dye solutions [3] and semiconductors [4] with randomly fluctuating dielectric constant as well as chaotic resonators with irregularly shaped boundaries [5]. Even in the absence of pumping random media show interesting interference effects such as coherent backscattering or localization of light [6]. The theoretical investigation of the quantum optical properties of random media started [7]8] with the demonstration of deviations from blackbody radiation in the case of one dimensional scattering. In a pioneering work [9] Beenakker generalized these results to multidimensional chaotic scattering by establishing a general relationship between the photocount statistics of a linear random medium and its scattering matrix. Using that relationship, Beenakker and coworkers predicted an excess photon noise due to multiple scattering [9], computed the photon noise power spectrum [10], and investigated the effect of photon localization on the photocount statistics [11].

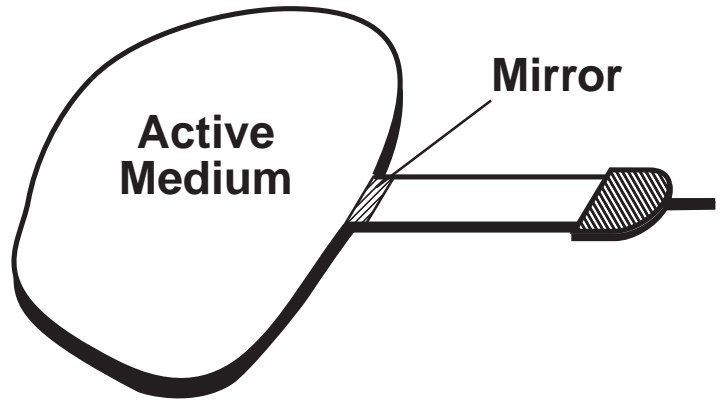

FIG. 1. Sketch of chaotic laser cavity with a partly transmitting mirror. The cavity is connected to a waveguide and a frequency selective photodetector.

In contrast to the wealth of results for linear random amplifiers, little is known about the photon statistics of random media above the lasing threshold. This is an important open problem since the experimental signatures of random lasing are still controversial [4]. In the present paper we address that problem for a chaotic laser resonator in the regime of single-mode lasing. We show that the chaotic nature of the cavity modes gives rise to fluctuations of the photocount on top of the quantum optical fluctuations known from laser theory. Chaos-induced fluctuations are found when a single-mode photodetection is performed over an ensemble of modes. The ensemble may be obtained from a single resonator upon varying suitable parameters or from different resonators with small variations in shape. The factorial moments of the photocount display strong fluctuations from one mode to another. However, we show that the probability density for each factorial moment depends only on general symmetries of the ensemble and on four parameters describing the laser dynamics; these are the coefficients of linear gain, mean escape loss, absorption loss and saturation of the amplifying medium.

A sketch of the system is shown in Fig. 1: A chaotic resonator with an irregularly shaped boundary is homogeneously filled with an active medium. A partly transmitting mirror reflects impinging radiation with mean probability $R$ back into the resonator. The fraction $T=1-R$ of radiation is transmitted and injected into a waveguide supporting $M$ transverse modes. A frequency selective photodetector counts the transmitted photons with efficiency 1 . We consider the case of a quasi discrete cavity spectrum; then the mean cavity escape rate $\bar{\Gamma}=M T \Delta \omega$ is smaller than the spacing $\Delta \omega$ of adjacent resonator modes. Accordingly, the mirror transmission coefficient $T$ must be small, $M T \ll 1$. The counting time $t$ will be 
assumed large enough that the radiation from individual cavity modes can be resolved, $t \Delta \omega \gg 1$.

As for the active medium, we allow for $N$ active atoms. The $\mu$ th atom "sees" the field mode through a coupling constant $g_{\mu}$ which is proportional to both the atomic dipole matrix element and the value of the lasing mode at the location of the atom. We only consider the simplest situation in which the characteristic times for atomic pump and losses are short compared to the mean life time of a photon in the cavity. The effect of the atoms on the field mode can then be represented by three parameters $\mathcal{A}, \mathcal{B}, \mathcal{C}$ that characterize the linear gain, the nonlinear saturation, and the total loss, respectively [2]. The first two of these depend on the coupling constants, $\mathcal{A} \sim \sum_{\mu=1}^{N} g_{\mu}^{2}, \mathcal{B} \sim \sum_{\mu=1}^{N} g_{\mu}^{4}$. Inasmuch as the resonator mode represents wave chaos, it varies quasi-randomly on the scale of the optical wavelength $\lambda[12,13$. Coupling constants $g_{\mu}$ associated with atoms separated by more than $\lambda$ therefore behave as independent random numbers. The coefficients $\mathcal{A}$ and $\mathcal{B}$, however, become sharp in the limit $N \gg 1$, due to the central limit theorem. The total loss rate $\mathcal{C}=\Gamma+\kappa$ is the sum of the photon escape rate $\Gamma$ and the absorption rate $\kappa$. Here, the absorption rate may be considered fixed while the escape rate is a random quantity, as will be revealed presently.

To calculate the photocount statistics we use the input-output theory of Gardiner and Collet [14,1. Waves entering and leaving the waveguide are described by $M$-component annihilation and creation operators $a^{\text {in }}, a^{\text {in } \dagger}, a^{\text {out }}, a^{\text {out } \dagger}$, which obey the commutation relations

$$
\left[a_{p}(t), a_{q}^{\dagger}\left(t^{\prime}\right)\right]=\delta_{p q} \delta\left(t-t^{\prime}\right), \quad\left[a_{p}(t), a_{q}\left(t^{\prime}\right)\right]=0 .
$$

Here, $p, q=1, \ldots, M$ label the transverse modes and $a=a^{\text {in }}$ or $a=a^{\text {out }}$. The boundary condition

$$
a_{p}^{\mathrm{in}}(t)+a_{p}^{\mathrm{out}}(t)=\gamma_{p} b(t)
$$

connects incoming and outgoing radiation in each transverse mode with the annihilation operator $b$ of the cavity mode. While the boundary condition (2) results from assuming a linear coupling between the waveguide and the cavity field, no restriction is imposed on the intracavity dynamics. Therefore, the input-output relation (2) holds both below and above threshold. The outcoupling amplitudes $\gamma_{p}$ must be considered independent random quantities with Gaussian statistics, since they represent the local fluctuations of the resonator mode across the outcoupling mirror. In fact, their randomness constitutes the principle effect of the wave chaos within the resonator on the ensemble fluctuations of the laser output.

In the linear regime below threshold, one can eliminate the cavity operators from Eq. (2) and express the outgoing radiation in terms of the incoming radiation, the intracavity noise, and the S-matrix [1] 9]. One thus obtains the photocount fluctuations [9] through the $\mathrm{S}-$ matrix statistics known for chaotic scattering.

We proceed to the nonlinear regime near and above threshold. For the case of a vacuum input, the boundary condition (2) allows to express all variances of the output field entirely in terms of the variances of the cavity field and the Gaussian statistics of the $\gamma_{p}$. We characterize the photocount statistics through the factorial moments

$$
\mu_{r}=\sum_{m=0}^{\infty} m(m-1) \cdots(m-r+1) p(m)
$$

of the probability $p(m)$ that $m$ photons are counted in a time interval $t$. That probability is given by [1,2]

$$
\begin{aligned}
p(m) & =\frac{1}{m !}\left\langle: W^{m} e^{-W}:\right\rangle \\
W & =\int_{0}^{t} d t^{\prime} \sum_{p=1}^{M} a_{p}^{\text {out } \dagger}\left(t^{\prime}\right) a_{p}^{\text {out }}\left(t^{\prime}\right) .
\end{aligned}
$$

Here $\langle\cdots\rangle$ denote the quantum steady-state average and the colons demand normal and a certain time ordering. Combining Eqs. (3)-(4) with the input-output relation (2) for a vacuum input we get

$$
\mu_{r}=\left\langle: W^{r}:\right\rangle=\Gamma^{r}\left\langle: I^{r}:\right\rangle
$$

where $\Gamma=\sum_{p=1}^{M}\left|\gamma_{p}\right|^{2}$ is the escape rate and $I^{r}$ the $r$ th power of the integrated cavity field intensity

$$
I=\int_{0}^{t} d t^{\prime} b^{\dagger}\left(t^{\prime}\right) b\left(t^{\prime}\right)
$$

Clearly, the first moment alias mean photocount, $\mu_{1}=$ $\Gamma t\left\langle b^{\dagger} b\right\rangle$, is a purely static quantity as it is proportional to the stationary mean photon number inside the cavity.

The factorial moments (5) specify the output statistics of a single cavity mode with the random escape rate $\Gamma$. The distribution of $\Gamma$ over an ensemble of modes is the $\chi_{\nu}^{2}$ distribution

$$
P(\Gamma)=A_{\nu} \Gamma^{\nu / 2-1} \exp (-\nu \Gamma / 2 \bar{\Gamma})
$$

well-known from random-matrix theory 15. Here, $\nu=$ $\beta M$ is an integer and $A_{\nu}$ a normalization constant. The value of the parameter $\beta$ depends on whether the system is time-reversal invariant $(\beta=1)$ or whether timereversal invariance is broken $(\beta=2)$ 15, 16. The special case $M=\beta=1$ is known as the Porter-Thomas distribution. Together with $\Gamma$ the factorial moments $\mu_{r}$ become random quantities. Their distribution is given by

$$
\mathcal{P}\left(\mu_{r}\right)=\int d \Gamma P(\Gamma) \delta\left(\mu_{r}-\Gamma^{r}\left\langle: I^{r}:\right\rangle\right) .
$$

Note that the right hand side involves a twofold average, the quantum optical average (represented by the brackets $\langle\cdots\rangle)$ and the ensemble average over the cavity modes 
(represented by the integral with the probability distribution $P(\Gamma)$ ). We emphasize that the quantum optical average $\left\langle: I^{r}:\right\rangle$ depends on $\Gamma$ through the total loss rate $\mathcal{C}$. We now discuss the result (8) in various limiting cases.

We first consider the case $M \gg 1$ of many transverse modes in the waveguide. The diameter of the waveguide is then much larger than the wavelength of the cavity mode. A simple saddle point argument shows that $P(\Gamma)$ for large $M$ approaches a Gaussian distribution with mean $\bar{\Gamma} \sim M$ and standard deviation $\Delta \Gamma=\bar{\Gamma} / \sqrt{\beta M / 2}$. The relative fluctuations are small, $\Delta \Gamma / \bar{\Gamma} \sim 1 / \sqrt{M} \rightarrow 0$ for $M \rightarrow \infty$, and the same is true for the fluctuations of all factorial moments. For cavities with large outcoupling mirrors we thus recover the sharp factorial moments one is used to from non-chaotic resonators.

Second, we investigate the limit of vanishing photocount, $\mu_{r} \rightarrow 0$. According to Eq. (5), this is the weakcoupling limit $\Gamma \rightarrow 0$ for which the photons in the cavity field can hardly escape into the waveguide. The laser dynamics becomes independent of the outcoupling loss as the total cavity loss $\mathcal{C}$ is fully dominated by the absorption loss. All cavity field moments become independent of $\Gamma$, and Eq. (5) reduces to $\mu_{r} \sim \Gamma^{r}$. Substitution into Eq. (8) yields the power-law behavior

$$
\mathcal{P}\left(\mu_{r}\right) \sim \mu_{r}^{(\beta M) /(2 r)-1},
$$

for $\mu_{r} \rightarrow 0$. A special case of this result is $M=\beta=1$ for which the distribution of the first and second moment diverge as $\mu_{1}^{-1 / 2}$ and $\mu_{2}^{-3 / 4}$ for $\mu_{1}, \mu_{2} \rightarrow 0$, respectively.

The third case is the short-time regime $t \ll t_{c}$ where $t_{c}$ is the correlation time of the intensity fluctuations of the cavity mode. During counting intervals that short the field intensity cannot vary appreciably. Therefore, the integrated intensity $I$ becomes the product of $t$ with the instantaneous photon number $\hat{n}=b^{\dagger} b$ in the cavity. The factorial moments of the output take the simple form

$$
\mu_{r}=(\Gamma t)^{r}\left\langle: \hat{n}^{r}:\right\rangle
$$

and one easily verifies that the normally ordered moment $\left\langle: \hat{n}^{r}:\right\rangle$ occuring here reduces to the $r$ th factorial moment of the stationary photon number distribution of the lasing mode. The latter has the form [1] 2]

$$
P_{n}=\mathcal{N} \frac{\left[\mathcal{A} n_{s} / \mathcal{C}\right]^{n+n_{s}}}{\left(n+n_{s}\right) !}
$$

Note that $P_{n}$ depends on the three laser parameters $\mathcal{A}$, $\mathcal{B}$, and $\mathcal{C}$; in particular, the nonlinearity $\mathcal{B}$ enters through the so-called saturation photon number $n_{s}=\mathcal{A} / \mathcal{B}$. The symbol $\mathcal{N}$ represents a normalization constant.

The calculation of the mean photocount $\mu_{1}$ reduces to a steady-state average for arbitrary counting time. We calculated $\mu_{1}$ numerically as a function of $\Gamma$ using the distribution (11). Substituting the result $\mu_{1}(\Gamma)$ into

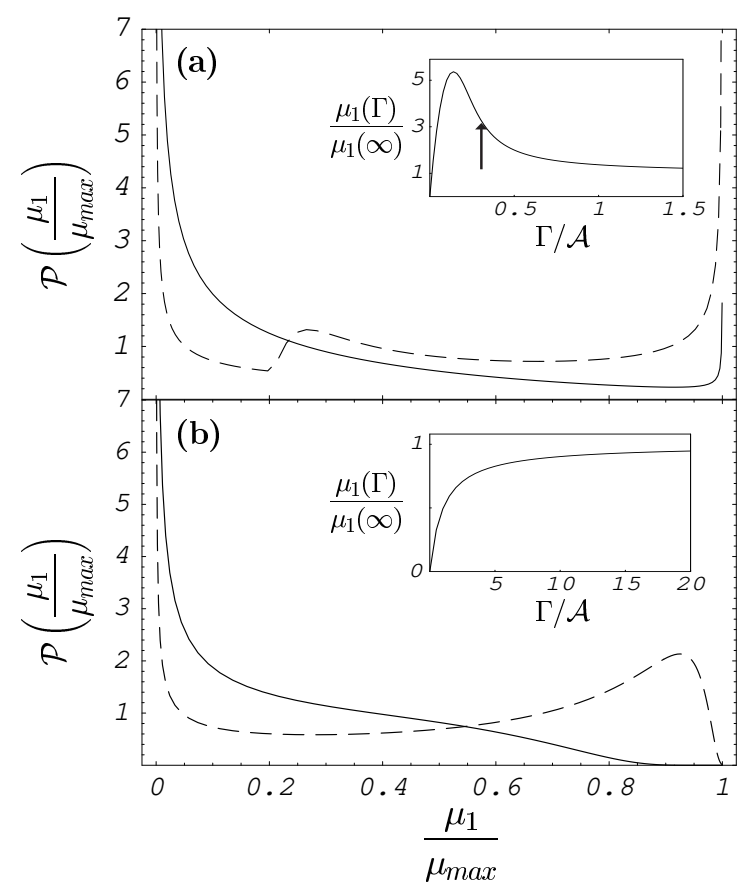

FIG. 2. Distribution $\mathcal{P}\left(\mu_{1} / \mu_{\max }\right)$ as a function of the dimensionless mean photocount $\mu_{1} / \mu_{\max }$ for $\beta M=1$ and four sets of laser parameters. Rates are given in units of $\mathcal{A} \equiv 1$, the nonlinearity is $\mathcal{B}=0.005$. (a) $\kappa=0.7, \bar{\Gamma}=0.02$ (solid line); $\kappa=0.7, \bar{\Gamma}=0.2$ (dashed line). (b) $\kappa=2.0, \bar{\Gamma}=0.5$ (solid line); $\kappa=2.0, \bar{\Gamma}=4.0$ (dashed line). The insets in (a) and (b) show $\mu_{1}$ as a function of $\Gamma / \mathcal{A}$. The arrow indicates the threshold-value of $\Gamma$ below which lasing takes place.

Eq. (8) and carrying out the integration over $\Gamma$, we obtained $\mathcal{P}\left(\mu_{1}\right)$. The results for time-reversal invariant cavities connected to a single-mode waveguide are plotted in Fig. 2, for $\beta M=1$ and four different sets of parameters. The two distributions in Fig. 2(a) correspond to $\mathcal{A}>\overline{\mathcal{C}}$, i.e. to lasers above threshold in the ensemble average. By contrast, $\mathcal{A}<\kappa$ for the distributions of Fig. 2(b); all lasers of those ensembles are below threshold irrespective of the escape rate $\Gamma$. We note that all distributions are strongly non-Gaussian. They are all peaked as $\mu_{1}^{-1 / 2}$ at small photocount, in accord with the general argument presented above for the asymptotics at $\mu_{1} \rightarrow 0$. Two further features spring to the eye and demand explanation: First, above threshold but not below we encounter an additional peak at maximum photocount; second, for certain cases (dashed lines in Fig. 2) the distribution $\mathcal{P}\left(\mu_{1}\right)$ displays a shoulder for sub-maximal $\mu_{1}$.

The origin of these structures lies in the $\Gamma$-dependence of the mean photocount $\mu_{1}$. That dependence is depicted in the insets and seen to be qualitatively different above and below threshold. While $\mu_{1}$ increases monotonically with $\Gamma$ in the below-threshold case, it develops a maximum at an intermediate value of $\Gamma$ when the laser is above threshold. This behavior can be understood from 
the simple analytic expressions

$$
\mu_{1} / t= \begin{cases}\Gamma n_{s} \frac{\mathcal{A}-\mathcal{C}}{\mathcal{C}} & \text { for } \mathcal{C}=\Gamma+\kappa<\mathcal{A} \\ \frac{\Gamma \mathcal{A}}{\mathcal{C}-\mathcal{A}} & \text { for } \mathcal{C}=\Gamma+\kappa>\mathcal{A}\end{cases}
$$

that follow from the photon number distribution (11) sufficiently far from threshold. According to Eq. (12), $\mu_{1}$ rises linearly with $\Gamma$ out of the origin and approaches an asymptotic plateau for very large $\Gamma$, as visible in Fig. 2. The rise to the plateau is monotonic when $\mathcal{A}<\kappa$ since the below-threshold case of $(12)$ applies for all values of $\Gamma$; the maximum photocount $\mu_{\max }$ is then just the plateau value. The laser of Fig. 2(a) is above threshold for small $\Gamma$ but below for large $\Gamma$. The maximum photocount $\mu_{\max }$ then arises for an intermediate value $\Gamma^{*}$. A simple argument can be employed to determine the border between the below- and the above-threshold ensemble. The argument follows from the observation that $\mu_{1}(\Gamma)$ approaches its plateau-value from above for the above-threshold ensemble while the plateau is approached from below when the ensemble is below threshold. From the lower case of Eq. (12), which becomes exact for $\Gamma \rightarrow \infty$, this yields the threshold condition $\mathcal{A}=\kappa$. To estimate the value of $\Gamma^{*}$ we may employ the above-threshold case of Eq. (12) and find $\Gamma^{*}=\sqrt{\mathcal{A} \kappa}-\kappa$.

Based on this understanding of $\mu_{1}(\Gamma)$ one can appreciate the above-threshold peak of $\mathcal{P}\left(\mu_{1}\right)$ at $\mu_{1}=\mu_{\text {max }}$. Substituting $\mu_{1}(\Gamma)=\mu_{\max }+\frac{1}{2} \mu^{\prime \prime}\left(\Gamma^{*}\right)\left(\Gamma-\Gamma^{*}\right)^{2}$ in the photocount distribution (8) we find that $\mathcal{P}\left(\mu_{1}\right)$ has a square-root singularity $\left|\mu_{1}-\mu_{\max }\right|^{-1 / 2}$ which is precisely the peak depicted in Fig. 2(a). Clearly, no such peak can arise in the below-threshold case of Fig. 2(b) as the photocount increases monotonically with $\Gamma$. Inspecting Eqs. (7), (8) in the limit $\Gamma \rightarrow \infty$ we rather find that $\mathcal{P}\left(\mu_{1}\right)$ vanishes when $\mu_{1}$ approaches $\mu_{\max }$.

The shoulders at sub-maximal photocount are caused by amplified spontaneous emission below the laser threshold. Formally, the shoulders arise from the asymptotic plateau of $\mu_{1}$ for large $\Gamma$. The definition (8) immediately implies enhanced probability for photocounts $\mu_{1}$ in the vicinity of that plateau. Note that the shoulder is invisible for the distribution shown as a solid line in Fig. 2(a), since for this distribution large values of $\Gamma$ are strongly suppressed by the small mean value $\bar{\Gamma}$. Further note that the shoulders for both curves in Fig. 2(b) lie closer to $\mu_{\max }$ than for the dashed curve in Fig. 2(a) since $\mu \rightarrow \mu_{\max }$ coincides with $\Gamma \rightarrow \infty$ in the regime below threshold.

In contrast to the mean photocount which can be expressed in terms of the stationary distribution of the laser, all $\mu_{r}$ with $r \geq 2$ involve dynamical information through correlation functions with $r-1$ time arguments. We defer the discussion of the higher factorial moments and their distribution to a separate publication 17 .

We finally compare our results with related fluctuation phenomena in other areas of physics and discuss possi- ble experimental tests. In nuclear physics the PortherThomas distribution describes level-width fluctuations in neutron scattering [15. The amplitude fluctuations of Coulomb blockade oscillations in semiconductor quantum dots are also of the Porther-Thomas type 18]. In both cases, as well as for the chaotic lasers studied in this paper, the fluctuations result from the chaotic nature of wave functions. However, in chaotic lasers new interesting features arise due to the interplay of wave chaos with the nonlinear dynamics of the laser. As a consequence of this interplay, the distribution of the mean photocount can strongly deviate from the Porter-Thomas distribution. To test the predicted mode-to-mode fluctuations experimentally, one must study the photocount statistics for an ensemble of chaotic modes. It seems feasible to generate such ensembles in tunable lasers e.g. by shape variations, or by the injection and displacement of artificial scatterers in the case of microwave cavities.

Support by the Sonderforschungsbereich "Unordnung und große Fluktuationen" der Deutschen Forschungsgemeinschaft is gratefully acknowledged.

[1] D. F. Walls and G. J. Milburn, Quantum Optics (Springer, Berlin, 1994).

[2] L. Mandel and E. Wolf, Optical Coherence and Quantum Optics (Cambridge University Press, Cambridge, 1995).

[3] M. N. Lawandy, R. M. Balachanfran, A. S. L. Gomes, and E. Sauvain, Nature (London) 368, 436 (1994).

[4] H. Cao, Y. G. Zhao, S. T. Ho, E. W. Seelig, Q. H. Wang, and R. P. H. Chang, Phys. Rev. Lett. 82, 2278 (1999).

[5] C. Gmachl, F. Capasso, E. E. Narimanov, J. U. Nöckel, A. D. Stone, J. Faist, D. L. Sivco, and A. Y. Cho, Science 280, 1556 (1998).

[6] Scattering and Localization of Classical Waves in Random Media, edited by P. Sheng (World Scientific, Singapore, 1990).

[7] T. Gruner and D.-G. Welsch, Phys. Rev. A 54, 1661 (1996).

[8] M. Artoni and R. Loudon, Phys. Rev. A 55, 1347 (1997).

[9] C. W. J. Beenakker, Phys. Rev. Lett. 81, 1829 (1998).

[10] E. G. Mishchenko, M. Patra, and C. W. J. Beenakker, cond-mat/0003262.

[11] C. W. J. Beenakker, M. Patra, and P. W. Brouwer, Phys. Rev. A 61, 051801(R) (2000).

[12] M. V. Berry, J. Phys. A 10, 2083 (1977).

[13] F. Haake Quantum Signatures of Chaos, 2nd edition (Springer, Berlin, 2000).

[14] C. W. Gardiner and M. J. Collet, Phys. Rev. A 31, 3761 (1985).

[15] T. Guhr, A. Müller-Groehling, and H. A. Weidenmüller, Phys. Rep. 299, 189 (1998).

[16] The case $\beta=2$ can be realized in microwave cavities containing magnetized ferrites.

[17] G. Hackenbroich, C. Viviescas, B. Elattari, and F. Haake, unpublished.

[18] R. A. Jalabert, A. D. Stone, and Y. Alhassid, Phys. Rev. Lett. 68, 3468 (1992). 